parathyroid hormone gene expression by hypocalcemia, hypercalcemia, and vitamin D in the rat. J. Clin. Invest. 86:1313-1319.

25. Naveh-Many, T., Friedlaender, M.M., Mayer, H., and Silver, J. 1989. Calcium regulates parathyroid hormone messenger ribonucleic acid (mRNA), but not calcitonin mRNA in vivo in the rat. Dominant role of 1,25-dihydroxyvitamin D. Endocrinology. 125:275-280.

26. Audran, M., Gross, M., and Kumar, R. 1986. The physiology of the vitamin D endocrine system. Semin. Nephrol. 6:4-20.

27. Audran, M., and Kumar, R. 1985. The physiology and pathophysiology of vitamin D. Mayo Clin. Proc. 60:851-866.

28. Cozzolino, M., et al. 2005. Pathogenesis of parathyroid hyperplasia in renal failure. J. Nephrol. 18:5-8.

29. Dusso, A.S., et al. 2007. Molecular basis of parathyroid hyperplasia. J. Ren. Nutr. 17:45-47.

30. Dusso, A.S., et al. 2006. Pathogenic mechanisms for parathyroid hyperplasia. Kidney Int. Suppl. 102:S8-S11.

31. Danese, M.D., et al. 2006. PTH and the risks for hip, vertebral, and pelvic fractures among patients on dialysis. Am. J. Kidney Dis. 47:149-156.

32. Jadoul, M., et al. 2006. Incidence and risk factors for hip or other bone fractures among hemodialysis patients in the Dialysis Outcomes and Practice Patterns Study. Kidney Int. 70:1358-1366.

33. Rudser, K.D., de Boer, I.H., Dooley, A., Young, B., and Kestenbaum, B. 2007. Fracture risk after parathyroidectomy among chronic hemodialysis patients. J. Am. Soc. Nephrol. 18:2401-2407.

34. Block, G.A., et al. 2004. Mineral metabolism, mortality, and morbidity in maintenance hemodialysis.
J. Am. Soc. Nephrol. 15:2208-2218.

35. Block, G.A., et al. 2004. Cinacalcet for secondary hyperparathyroidism in patients receiving hemodialysis. N. Engl. J. Med. 350:1516-1525.

36. Drueke, T.B. 2004. Calcimimetics versus vitamin D: what are their relative roles? Blood Purif. 22:38-43.

37. Locatelli, F., et al. 2002. Management of disturbances of calcium and phosphate metabolism in chronic renal insufficiency, with emphasis on the control of hyperphosphataemia. Nephrol. Dial. Transplant. 17:723-731.

38. Moe, S.M., and Drueke, T.B. 2003. Management of secondary hyperparathyroidism: the importance and the challenge of controlling parathyroid hormone levels without elevating calcium, phosphorus, and calcium-phosphorus product. Am. J. Nephrol. 23:369-379.

39. Moallem, E., Kilav, R., Silver, J., and Naveh-Many, T. 1998. RNA-Protein binding and post-transcriptional regulation of parathyroid hormone gene expression by calcium and phosphate. J. Biol. Chem. 273:5253-5259.

40. Levi, R, et al. 2006. Increased parathyroid hormone gene expression in secondary hyperparathyroidism of experimental uremia is reversed by calcimimetics: correlation with posttranslational modification of the trans acting factor AUF1. J. Am. Soc. Nephrol. 17:107-112.

41. Naveh-Many, T., Sela-Brown, A., and Silver, J. 1999. Protein-RNA interactions in the regulation of PTH gene expression by calcium and phosphate. Nephrol. Dial. Transplant. 14:811-813.

42. Kilav, R., Bell, O., Le, S.Y., Silver, J., and Naveh-Many, T. 2004. The parathyroid hormone mRNA 3'-untranslated region AU-rich element is an unstructured func- tional element. J. Biol. Chem. 279:2109-2116.

43. Naveh-Many, T., Bell, O., Silver, J., and Kilav, R. 2002. Cis and trans acting factors in the regulation of parathyroid hormone (PTH) mRNA stability by calcium and phosphate. FEBS Lett. 529:60-64.

44. Nechama, M., Ben-Dov, I.Z., Briata, P., Gherzi, R., and Naveh-Many, T. 2008. The mRNA decay promoting factor K-homology splicing regulator protein post-transcriptionally determines parathyroid hormone mRNA levels. FASEB J. 22:3458-3468.

45. Nechama, M., Ben-Dov, I.Z., Silver, J., and NavehMany, T. 2009. Regulation of PTH mRNA stability by the calcimimetic R568 and the phosphorus binder lanthanum carbonate in CKD. Am. J. Physiol. Renal Physiol. 296:F795-F800.

46. Nechama, M., Uchida, T., Mor Yosef-Levi, I., Silver, J., and Naveh-Many, T. 2009. The peptidyl-prolyl isomerase Pin1 determines parathyroid hormone mRNA levels and stability in rat models of secondary hyperparathyroidism. J. Clin. Invest. 119:3102-3114.

47. Lu, K.P., Liou, Y.C., and Zhou, X.Z. 2002. Pinning down proline-directed phosphorylation signaling. Trends Cell Biol. 12:164-172.

48. Lu, K.P., and Zhou, X.Z. 2007. The prolyl isomerase PIN1: a pivotal new twist in phosphorylation signalling and disease. Nat. Rev. Mol. Cell Biol. 8:904-916.

49. Shen, Z.J., Esnault, S., and Malter, J.S. 2005. The peptidyl-prolyl isomerase Pin1 regulates the stability of granulocyte-macrophage colony-stimulating factor mRNA in activated eosinophils. Nat. Immunol. 6:1280-1287.

50. Shen, Z.J., et al. 2008. Pin 1 regulates TGF-beta1 production by activated human and murine eosinophils and contributes to allergic lung fibrosis. J. Clin. Invest. 118:479-490.

\title{
Resolving lung injury: a new role for Tregs in controlling the innate immune response
}

\author{
Anthony Pietropaoli and Steve N. Georas
}

Division of Pulmonary and Critical Care Medicine, University of Rochester Medical Center, Rochester, New York, USA.

\begin{abstract}
Inflammation-associated lung injury is a major cause of morbidity and mortality for patients in intensive care units. Although the cellular and molecular events that initiate lung inflammation are now well understood, the mechanisms that promote its resolution remain poorly defined. In this issue of the JCI, D'Alessio et al. show in a mouse model that recovery from acute lung injury is not simply a passive process, but involves Tregs in an active resolution program (see the related article beginning on page 2898).
\end{abstract}

\section{Overview of lung injury}

Acute lung injury (ALI) is a syndrome defined by bilateral pulmonary infiltrates on chest radiography and arterial hypoxemia in the absence of left atrial hypertension. This syndrome has a rapidly progressive clinical presentation characterized by acute onset of dyspnea and respiratory fail-

Conflict of interest: The authors have declared that no conflict of interest exists.

Citation for this article: J. Clin. Invest. 119:2891-2894 (2009). doi:10.1172/JCI40880. ure requiring mechanical ventilation; when arterial hypoxemia is particularly severe, it is classified as acute respiratory distress syndrome (ARDS; ref. 1). ALI can be caused by direct and indirect pulmonary insults. The initial pathophysiologic events are endothelial and epithelial injury, excessive permeability of the alveolar-capillary unit, and alveolar and interstitial accumulation of proteinaceous fluid and inflammatory cells (1). This inflammatory response disrupts alveolar function, impairing fluid clearance, surfactant production, and gas exchange (2). Neutrophils are a key component of lung inflammation in ALI and are recruited to the lung by chemotactic signals produced by alveolar macrophages and other lung cell types $(3,4)$.

ALI occurs in response to a variety of acute illnesses, with common direct causes including pneumonia and aspiration of gastric contents, and common indirect causes including extrapulmonary sepsis syndrome and trauma $(5,6)$. The public health burden is high, with an incidence of approximately 80 cases per 100,000 person-years and mortality approaching $40 \%$ (7). Interestingly, persistent pulmonary inflammation and dysfunction are strongly associated with mortality (8), yet the cause of death in patients with ALI is usually multisystem organ failure instead of intractable respiratory insufficiency (9). This apparent paradox is explained by 
Table 1

Other potential antiinflammatory or proresolution mediators and molecules in the lung

Secreted, soluble, or gaseous mediators

Carbon monoxide

Catecholamines

Endocannabinoids

Indoleamine 2,3 dioxygenase

NO, NO metabolites, and NO-regulated proteins

Soluble IL-6 receptor (sIL6R)

Surfactant proteins and related phospholipids

\section{Cell surface receptors}

Adenosine receptor 2B (A2BAR)

Annexin 1

Inhibitory costimulatory molecules (e.g., B and T lymphocyte attenuator [BTLA]

and programmed cell death 1 [PD-1])

Mucin 1 (MUC1)

Intracellular molecules or transcription factors

Aryl hydrocarbon receptor (AhR)

Hematopoietic prostaglandin D2 synthase (hPGD2S)

Heme oxygenase 1 (HO-1)

NOS

PPAR $\gamma$

Mediators and molecules are in addition to those described in the text. This table is not meant to be exhaustive.

animal and human studies showing that inflammatory mediators from the lung spill into the systemic circulation, inducing extrapulmonary organ dysfunction and promoting the downhill cascade toward multisystem organ failure (10). Therefore, therapies aimed specifically at accelerating resolution of the pulmonary inflammatory response could pay dividends that extend well beyond the lung. Over the last decade, it has been proven that reducing ventilator-delivered pressure and volume attenuate pulmonary and systemic inflammation and mortality (11), but pharmacologic therapies for ALI remain limited. Glucocorticoids have been extensively studied, but their efficacy remains controversial (12), and the search for other effective antiinflammatory treatments continues.

\section{Resolution biology: an emerging discipline}

Although inflammation is an essential and protective response to tissue injury and infection, excessive and persistent inflammation can lead to chronic organ dysfunction. In the case of lung injury, unchecked inflammation likely contributes to the progression from ALI to ARDS to multisystem organ failure. Therefore, once the offending insult has been neutralized, prompt resolution of lung inflammation is a desirable goal. Why this does not occur in many patients is currently unclear; in fact, the mechanisms involved in the resolution of inflammation in general are still poorly understood. In their study reported in this issue of the JCI, D'Alessio et al. have addressed this gap in our current knowledge and firmly implicate a versatile subset of $\mathrm{CD}^{+} \mathrm{T}$ lymphocytes in the resolution of ALI in mice exposed to LPS (13).

In many models, resolution of inflammation is not simply a passive process (e.g., caused by removal of the offending insult and burnout of inflammatory cells), but involves active resolution programs (14). New cellular and molecular pathways involved in the resolution of inflammation are being uncovered at a rapid pace (Table 1), and although the precise distinction between proresolution and antiinflammatory can be challenging, recent working definitions have been proposed that should facilitate future research in this area. For example, a careful kinetic analysis of inflammatory cell numbers can define the resolution interval (Ri), the time from maximal infiltration of inflammatory cells to the $50 \%$ reduction point (15). Resolution-promoting pathways or molecules should be particularly effective at reducing the Ri and may have less effect on the magnitude or extent of inflammation at its peak. Proresolution mediators might also preferentially accumulate or be generated at later time points during the course of an inflammatory reaction. Although the Ri has not been extensively studied in human subjects, support for the importance of prompt resolution comes from the observation that BAL neutrophils remain elevated in nonsurvivors with ARDS (3).

During the resolution phase, recruited cells and necrotic material need to be cleared and disrupted tissue structures regenerated. To use a military analogy, after the troops have been marshaled and the enemy neutralized, the battlefield needs to be cleaned up. In ALI, neutrophils need to be cleared from the alveolar space, endothelial and epithelial barrier structures regenerated, and edema fluid reabsorbed (2). Neutrophil apoptosis is an important first step in the transition to resolution, because apoptosis preserves membrane structure and results in noninflammatory cell death and clearance. Interestingly, engulfment of apoptotic neutrophils by macrophages (via recognition of cell surface phosphatidylserine and other surface receptors) induces a phenotypic switch away from proinflammatory cytokine production and toward secretion of proresolution mediators such as TGF- $\beta$ (16). The regulation of macrophage phenotype may also involve additional cellular and soluble inputs. For example, clearance of apoptotic cells (i.e., efferocytosis) can be inhibited by factors secreted by immune cells (17). Perhaps the persistence of BAL neutrophils in nonsurvivors with ARDS reflects a failure to undergo apoptosis and efferocytosis, undermining the proresolution program?

An important recent advance came from the discovery of novel lipid mediators that are generated late in inflammatory reactions and promote active resolution of inflammation by inhibiting granulocyte infiltration and promoting monocyte recruitment. These include metabolites of arachidonic acid (e.g., lipoxin A4 and aspirin-triggered 15 epi-lipoxins) and omega-3 polyunsaturated fatty acids (e.g., resolvins, protectins, and the recently described maresins; refs. 14, 18). This is an active area of research in which new metabolic pathways, cell surface receptors, and therapeutic potentials are being rapidly defined. For example, resolvin E1 was recently shown to markedly hasten the resolution of allergic airway inflammation in a mouse model, in part by enhancing the clearance (or reducing the Ri) of different inflammatory cell types (19). Using a model similar to that of the 
A Normal lung

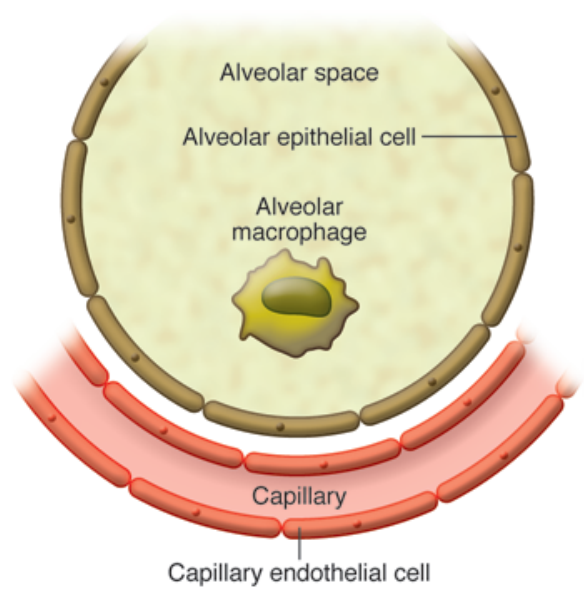

B ALI LPS,

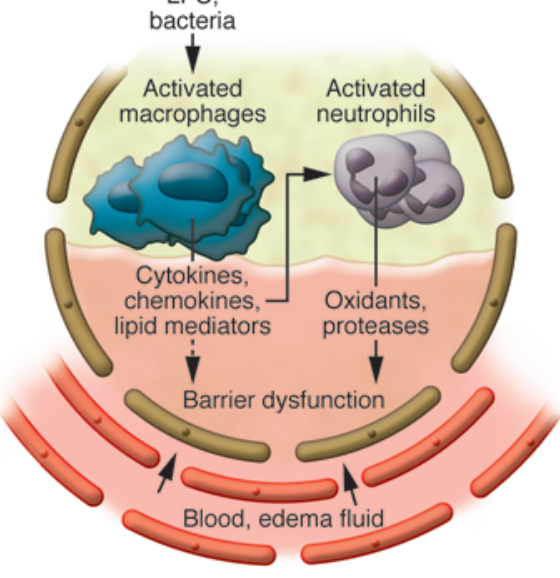

C Resolution phase

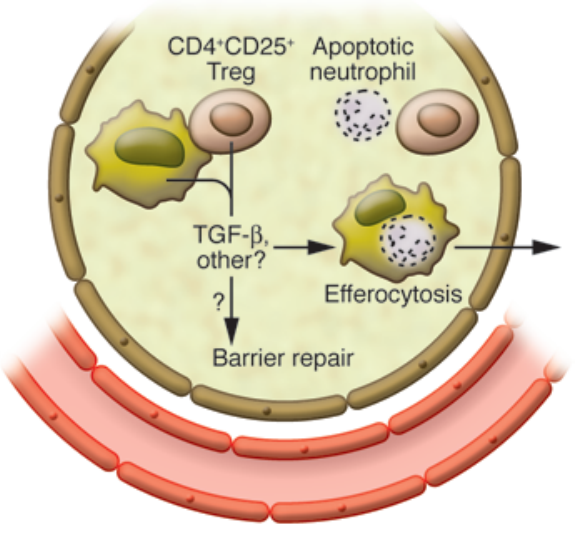

Figure 1

Potential mechanisms by which Tregs suppress inflammation and lung injury. In the normal lung (A), only alveolar macrophages are present within the distal air spaces. After LPS-induced ALI (B), neutrophils and macrophages accumulate in the alveolar space and release inflammatory mediators that cause barrier dysfunction and pulmonary edema. In their study in mice in this issue of the $\mathrm{JCl}$, D'Alessio et al. (13) report that effective resolution of this process involves $C D 4{ }^{+}$CD25+ Tregs $(\mathbf{C})$, which cause neutrophil apoptosis and efferocytosis and produce the proresolution mediator TGF- $\beta$ upon contact with alveolar macrophages.

D’Alessio et al. study (13), aspirin-triggered 15 epi-lipoxin A4 was also shown to attenuate LPS-induced lung injury in mice by countering myeloperoxidase signaling (20). Other lipid compounds, including lysolipid and sphingolipid mediators (e.g., lysophosphatidic acid and sphingosine-1 phosphate), have potential antiinflammatory and proresolution effects because they promote cellular egress from tissue and tighten barrier structures (21). Very little is known about the expression or function of these novel lipid mediators in humans with ALI or ARDS. Although they are challenging to measure in biological fluids, future studies investigating their expression and function in patients with different stages and severity of lung injury may be enlightening.

\section{Resolution of lung injury: a new role for Tregs}

D'Alessio et al. used multiple approaches, including adoptive transfer and neutralizing antibodies, to uncover a crucial role for $\mathrm{CD}^{+} \mathrm{CD} 25^{+} \mathrm{Foxp}^{+}$Tregs in resolving LPSinduced lung inflammation in mice (13). This important study establishes what we believe to be a new player in the resolution program and implicates a versatile subset of antigen-specific $\mathrm{CD}^{+} \mathrm{T}$ lymphocytes in suppressing an innate immune response that is not classically considered to be antigen driven. Similar to conventional T cells, $\mathrm{CD}^{+} \mathrm{CD} 25^{+} \mathrm{Foxp}^{+}$Tregs develop in the thymus and escape into the periphery with a diverse repertoire of TCRs that recognize both foreign and self peptides (although probably enriched in the latter). The vast majority of research to date has implicated Tregs in suppressing antigen-driven and $\mathrm{T}$ cell-dependent adaptive immune responses, especially in models of autoimmunity, infections, and cancer (reviewed in refs. 22, 23). In these diseases, Tregs are thought to be activated in an antigen-dependent manner and to inhibit self-reactive or pathogenic $\mathrm{T}$ cells through multiple mechanisms, including contact-dependent inhibitory cell surface receptors (e.g., CTLA-4), secretion of inhibitory cytokines (e.g., IL-10 and TGF- $\beta$ ), competition for growth factors (e.g., IL-2), and potentially direct lysis (24).

In contrast to these models, LPS-induced ALI occurs independently of antigen-activated lymphocytes because of nonspecific inflammation induced by innate immune cells expressing the TLR4 signaling complex (e.g., macrophages, epithelial cells, and dendritic cells). In support of this notion, D'Alessio et al. found no differences in early inflammatory indices in RAG-1-deficient mice, which lack mature $T$ and $B$ cells, and in fact found that these immunodeficient mice fared substantially worse at later time points after LPS challenge, with markedly enhanced lung inflammation (13). By performing careful kinetic analyses, flow cytometry, and adoptive transfer studies, these authors convincingly demonstrated that $\mathrm{CD} 4^{+} \mathrm{CD} 25^{+} \mathrm{T}$ cells expressing the Treg markers Foxp3 and folate receptor 4 were crucially involved in resolving lung injury and inflammation at these later time points. Importantly, CD $4^{+} \mathrm{CD} 25^{+}$Tregs were still effective when administered 24 hours after LPS, in contrast to most studies, which test agents administered before the onset of lung injury. The authors' findings add to a growing body of literature implicating $\mathrm{T}$ cells (both conventional and regulatory) in dampening innate immune responses that are typically independent of a specific antigen (25-27).

By measuring and neutralizing cytokines and performing in vitro coculture assays, D'Alessio et al. (13) implicate a model in which Tregs in the alveolar space induce neutrophil apoptosis and suppress macrophage cytokine secretion independent of the antiinflammatory cytokine IL-10, and dependent at least in part on TGF- $\beta$ (Figure 1). The authors show that TGF- $\beta$ is produced during cell-cell interactions between Tregs and macrophages in vitro (in keeping with current models of TGF- $\beta$ production; ref. 28), possibly reflecting TLR4 engagement on both cell types (29). However, it seems unlikely that sufficient LPS persists in the lung at later time points after challenge (e.g., 7-10 days) to engage TLRs expressed on alveolar Tregs. Another possibility is that the intense inflammation accompanying LPS challenge enhances antigen presentation within the 
lung and that $\mathrm{CD} 4{ }^{+} \mathrm{CD} 25^{+} \mathrm{Foxp}^{+}$Tregs are indeed activated in an antigen-dependent manner. Future studies examining the TCR specificity and activation of Tregs - and conventional $\mathrm{T}$ cells - in the lung during ALI should be revealing. Perhaps, in this context, we should refer to this remarkably versatile resolution-promoting cell type as Tres?

\section{Future directions and translational perspectives}

The report by D'Alessio et al. (13) raises some important questions and should spur future research in the field. First, what are the mechanisms by which $\mathrm{CD} 4{ }^{+} \mathrm{CD} 25^{+}$Tregs accumulate in the lung at later stages of lung injury? The authors consider both recruitment of cells into the alveolar space from the circulation and expansion from resident lung lymphocytes, possibilities that are not mutually exclusive. It will be interesting in future studies to determine the role of chemokines and cytokines (e.g., IL-2 and others that signal through the common $\gamma$ chain) in Treg expansion in the lung. Future studies using transgenic and gene-targeted mice in which Foxp $3^{+}$Tregs can be tracked and deleted may be particularly insightful in this regard and may avoid some of the artifacts introduced by adoptive transfer. Second, are other pro-resolution mediators produced during interactions between Tregs and lung cells (e.g., some of the lipid mediators discussed above)? In addition to hastening clearance of inflammatory cells, do Tregs or proresolution mediators accelerate the recovery of epithelial and endothelial barrier function? Third, would a similar protective effect of Tregs be observed in models of lung injury induced by active infections in which antigen-specific lymphocytes may be required to help clear the pathogens? Most importantly, D'Alessio et al. have identified a cell type involved in the resolution of ALI that we should now start studying in detail in human subjects.

\section{Acknowledgments}

The authors acknowledge grant support from the NIH (K23HL080077, to A. Pietropaoli; R01HL071933, to S.N. Georas), University of Rochester Clinical and Translational Sciences Institute, and University of Rochester Medical Center Department of Medicine.

Address correspondence to: Steve N. Georas or Anthony Pietropaoli, Division of Pulmonary and Critical Care Medicine, University of Rochester Medical Center, Rochester, New York 14642, USA. Phone: (585) 2754861; Fax: (585) 275-1171. E-mail: Steve_ Georas@urmc.rochester.edu (S.N. Georas); Anthony_Pietropaoli@urmc.rochester.edu (A. Pietropaoli).

1. Ware, L.B., and Matthay, M.A. 2000. The acute respiratory distress syndrome. N. Engl. J. Med. 342:1334-1349.

2. Matthay, M.A., and Wiener-Kronish, J.P. 1990. Intact epithelial barrier function is critical for the resolution of alveolar edema in humans. Am. Rev. Respir. Dis. 142:1250-1257.

3. Steinberg, K.P., et al. 1994. Evolution of bron choalveolar cell populations in the adult respiratory distress syndrome. Am. J. Respir. Crit. Care Med. 150:113-122.

4. Pittet, J.F., Mackersie, R.C., Martin, T.R., and Matthay, M.A. 1997. Biological markers of acute lung injury: prognostic and pathogenetic significance. Am. J. Respir. Crit. Care Med. 155:1187-1205.

5. Bernard, G.R., et al. 1994. The American-European Consensus Conference on ARDS. Definitions, mechanisms, relevant outcomes, and clinical trial coordination. Am.J. Respir. Crit. Care Med. 149:818-824.

6. Rubenfeld, G.D., and Herridge, M.S. 2007. Epidemiology and outcomes of acute lung injury. Chest. 131:554-562.

7. Rubenfeld, G.D., et al. 2005. Incidence and outcomes of acute lung injury. N. Engl. J. Med. 353:1685-1693.

8. Meduri, G.U., et al. 1995. Inflammatory cytokines in the BAL of patients with ARDS. Persistent elevation over time predicts poor outcome. Chest. 108:1303-1314.

9. Stapleton, R.D., et al. 2005. Causes and timing of death in patients with ARDS. Chest. 128:525-532.

10. Ranieri, V.M., et al. 1999. Effect of mechanical ventilation on inflammatory mediators in patients with acute respiratory distress syndrome: a randomized controlled trial. JAMA. 282:54-61.

11. [No authors listed.] 2000. Ventilation with lower tidal volumes as compared with traditional tidal volumes for acute lung injury and the acute respiratory distress syndrome. The Acute Respiratory Distress Syndrome Network. N. Engl. J. Med.
342:1301-1308.

12. Tang, B.M., Craig, J.C., Eslick, G.D., Seppelt, I., and McLean, A.S. 2009. Use of corticosteroids in acute lung injury and acute respiratory distress syndrome: a systematic review and meta-analysis. Crit. Care Med. 37:1594-1603.

13. D'Alessio, F.R., et al. 2009. CD $4^{+} \mathrm{CD} 25^{+}$Foxp $3^{+}$ Tregs resolve experimental lung injury in mice and are present in humans with acute lung injury. J. Clin. Invest. 119:2898-2913.

14. Serhan, C.N., Chiang, N., and Van Dyke, T.E. 2008. Resolving inflammation: dual anti-inflammatory and pro-resolution lipid mediators. Nat. Rev. Immunol. 8:349-361.

15. Serhan, C.N., et al. 2007. Resolution of inflammation: state of the art, definitions and terms. FASEB J. 21:325-332.

16. Huynh, M.L., Fadok, V.A., and Henson, P.M. 2002. Phosphatidylserine-dependent ingestion of apoptotic cells promotes TGF- $\beta 1$ secretion and the resolution of inflammation. J. Clin. Invest. 109:41-50.

17. Liu, G., et al. 2008. High mobility group protein-1 inhibits phagocytosis of apoptotic neutrophils through binding to phosphatidylserine. J. Immunol. 181:4240-4246.

18. Serhan, C.N., et al. 2009. Maresins: novel macrophage mediators with potent antiinflammatory and proresolving actions. J. Exp. Med. 206:15-23.

19. Haworth, O., Cernadas, M., Yang, R., Serhan, C.N., and Levy, B.D. 2008. Resolvin E1 regulates interleukin 23, interferon-gamma and lipoxin A4 to promote the resolution of allergic airway inflammation. Nat. Immunol. 9:873-879.

20. El Kebir, D., et al. 2009. 15-epi-lipoxin A4 inhibits myeloperoxidase signaling and enhances resolution of acute lung injury. Am. J. Respir. Crit. Care Med. 180:311-319.

21. Peng, X., et al. 2004. Protective effects of sphingosine 1-phosphate in murine endotoxin-induced inflammatory lung injury. Am. J. Respir. Crit. Care Med. 169:1245-1251.

22. Fehervari, Z., and Sakaguchi, S. 2004. CD4+ Tregs and immune control. J. Clin. Invest. 114:1209-1217.

23. Lu, L.F., and Rudensky, A. 2009. Molecular orchestration of differentiation and function of regulatory T cells. Genes Dev. 23:1270-1282.

24. Sojka, D.K., Huang, Y.H., and Fowell, D.J. 2008. Mechanisms of regulatory T-cell suppression - a diverse arsenal for a moving target. Immunology. 124:13-22.

25. Maloy, K.J., et al. 2003. CD4+CD25+ T(R) cells suppress innate immune pathology through cytokinedependent mechanisms. J. Exp. Med. 197:111-119.

26. Kinsey, G.R., et al. 2009. Regulatory T cells suppress innate immunity in kidney ischemia-reperfusion injury. J. Am. Soc. Nephrol. 20:1744-1753.

27. Guarda, G., et al. 2009. T cells dampen innate immune responses through inhibition of NLRP1 and NLRP3 inflammasomes. Nature. 460:269-273.

28. Li, M.O., and Flavell, R.A. 2008. TGF-beta: a master of all T cell trades. Cell. 134:392-404.

29. Caramalho, I., et al. 2003. Regulatory T cells selectively express toll-like receptors and are activated by lipopolysaccharide. J. Exp. Med. 197:403-411. 